RAD Conference Proceedings, vol. 4, pp. 106-108, 2020

ISSN 2466-4626 (online) | DOI: 10.21175/RadProc.2020.22

www.rad-proceedings.org

\title{
CHEMICAL EFFECTS OF NEUTRON IRRADIATION ON ARSENIC SULFIDES
}

\author{
J. F. Facetti-Masulli*, H. D. Colman
}

Universidad Nacional de Asunción \& Comisión Nacional de Energía Atómica, Asunción, Paraguay

\begin{abstract}
The distribution of valence states III and $V$ of radioactive ${ }^{76} A s$ after neutron irradiations of thioarsenic compounds has been investigated in arsenic sulphides, optical glasses materials. Compounds were irradiated with neutrons at a flux of $10^{12} \mathrm{n} \mathrm{cm}^{-2} \mathrm{~s}^{-1}$. The radioisotope formed by $(n, \gamma)$ reaction is ${ }^{6} \mathrm{As}$ with $T_{1 / 2}=26.4 \mathrm{~h}$. Separations of valence states were performed by electrophoresis. Radiochemical yields of As $V$ were higher than those obtained by other workers in the irradiation of arsenic oxygenated compounds; a similar high yield of $S b V$ was also found previously on the irradiation of thioantimony compounds and seems to be related to the covalent character of the As-S bond; likewise, internal conversion accounts for the primary oxidation of recoil atoms.
\end{abstract}

Key words: Radiative neutron capture, chemical consequences, arsenic thiocompounds, short range order, valence states distribution

\section{INTRODUCTION}

Arsenic and antimony thio-compounds as well as other chalcogenides are important components/materials of optical glasses according to their optical electronic properties [1-5]. They are part of materials that due to their particular electronic/bonding characteristics, the values of certain physical properties make them different from solids with covalent or metallic bonding; further from the former, closer to the latter [6]. Under neutron bombardment they should present changes in such properties as it happens, at very much lesser extent, when they are submitted inter alia to light or thermal induced processes [7]; and generate, therefore, chemical effects on the compounds.

Several studies have been performed on the distribution of the activities formed after neutron irradiation of arsenic and antimony oxicompounds [813], but on chalcogenide compounds, very few of such studies were made $[14,15]$.

Under irradiation, in arsenic oxycompounds internal conversion of the neutron capture gamma rays should result in oxidation of *As [8], but this was not observed, and electron transfer could have occurred in the solid state or in dissolution. In irradiated antimony oxides, the distribution of valence states indicated a much lower yield of ${ }^{*} \mathrm{SbV}$ [9] in comparison with the values of ${ }^{*} \mathrm{AsV}$ obtained on irradiating arsenic oxides $[8,9]$; nevertheless the yield of $* \mathrm{AsV}$ on the irradiation of $\mathrm{As}_{2} \mathrm{O}_{3}$ was low [10]. In regard to antimony thiocompounds bombarded with neutrons, the distribution of the valence states of radioactive atoms of antimony, was studied and compared with that found upon neutron irradiation of its oxygenated compounds. A higher yield of the Sb pentavalent state was found for the sulphides as well as for the hydrated compounds. This effect was considered to be related to the covalent character of the $\mathrm{Sb}-\mathrm{S}$ bond, to the degree of hydration of the compound and to the defects introduced in the lattice upon irradiation [13-15].

The present work consists of a comparative study of the distribution of activity of As-III and As V formed on irradiating tri and penta arsenic sulfides.

\section{EXPERIMENTAL}

\subsection{Materials}

Materials activated were amorphous [16] analytical grade As2S3 and As2S5 used without further purification.

\subsection{Irradiations}

Materials were irradiated in a nuclear reactor with flux of $2 \mathrm{x}^{1 \mathrm{O}^{12}} \mathrm{n} \mathrm{cm}^{2} \mathrm{seg}^{-1}$ under nitrogen or in evacuated ampoules. When arsenic compounds are irradiated with neutrons, the radioisotope ${ }^{76} \mathrm{As}$ of known characteristics [17], is formed by ( $\mathrm{n} \gamma$ ) reaction; ${ }^{76} \mathrm{As}$ by $\beta$ - decay $\left(\mathrm{T}_{1 / 2}=26.4 \mathrm{~h}\right)$ originates stable ${ }^{76} \mathrm{Se}$.

\subsection{Analysis}

The activated compounds were dissolved in $4.3 \%$ sodium sulfide solution in the presence of either, AsIII or As V carrier. The various valence states were separated by paper electrophoresis [18] using Whatman $3 \mathrm{MM}$ paper. The voltage gradient was $40 \mathrm{~V} / \mathrm{cm}$ and the electrolyte was a dissolution of $0.4 \%$ in $\mathrm{Na}_{2} \mathrm{~S}$ and $0.04 \%$ in $\mathrm{NaOH}$.

*jfrafama@rieder.net.py 


\subsection{Measurements}

The counting of the paper strips of $1 \mathrm{~cm}$ wide was carried out either with an end window GM counter or with a $\mathrm{NaI}(\mathrm{Tl})$ crystal.

\section{RESULTS AND DISCUSSION}

The distribution of valence states III \& V of radioactive arsenic according their activities is breakdown in Table 1.

Table 1. Distribution of *AsIII \& *AsV

\begin{tabular}{|l|l|l|c|}
\hline Compounds & As III \% & As V \% & Experiments (n) \\
\hline As2 33 & $28.2 \pm 3.4$ & $71.0 \pm 3.8$ & $(8)$ \\
\hline As2 55 & $1.0 \pm 0.9$ & $99.0 \pm 0.9$ & $(5)$ \\
\hline
\end{tabular}

Arsenic trisulfide As2S3, natural minerals orpiment \& anorpiment, have layered structures [19] of As and S atoms bonded with covalent interatomic forces, and the interlayers, by Vander Waals bonding that origenates a very stable high packing of layers [1]. The amorphous variety is constituted by clusters, i.e. microstructural units responsabile of the short/middle range order $[4,20]$.

In regard to the pentasulfide $\mathrm{As}_{2} \mathrm{~S}_{5}$, its structure is unknown though from the study of amorphous $\mathrm{As}_{2} \mathrm{~S}_{5}$ it is known that the short/middle range order is similar to that of amorphous $\mathrm{As}_{2} \mathrm{~S}_{3}$, but the structure of $\mathrm{As}_{2} \mathrm{~S}_{5}$ is more rigid [20]. According to ref [4] by quantum chem. calculations some cluster can be identified (are presented) as As2S5, As4 46 , As6S9.

Under irradiation (see Table 1), the radiochemical yield here obtained of AsV was higher in the thiocompounds than in the oxides and as in the case of neutron activated antimony sulfides, this higher yield of pentavalent atoms can be attributed to the type of structure, to the covalent character of As-S, electronegativity difference $\Delta \mathrm{X}=0.5$; the energy difference of levels $3 \mathrm{p}-3 \mathrm{~d}=101.2 \mathrm{eV}$ and for the state $\mathrm{s}^{1} \mathrm{p}^{3}$ is $102.3 \mathrm{eV}$, easily overlapping the electronic levels [21].

In the nuclear process ${ }^{75} \mathrm{As}(\mathrm{n}, \gamma){ }^{76} \mathrm{As}$ the prompt $\gamma$ spectrum is very complex and broad; the number of gamma being $\mathrm{N} \gamma=348$ with a wide de-excitation energy photon distribution: extended from 44.42 to 7284.07 KeV [22]; most intense of 559.10, 165.049, $86.788 \mathrm{KeV}$. Then, the recoil atoms energy spectrum must also be broad and most of them can not travel far in the solid before resting, only a few inter atomic, lattice distances. The more energetics, transfer their energy into the lattice and produce the ejection of atoms [23] in the chalcogenide structure originating cationic vacancies "incipient $\mathrm{V}$ centres" but most of them producing in the host atoms electronic excitation, vacancy cascades, ionizations as well as changes in the sulfides bandgap.

The lapse evolved from the very instant of the nuclear event $\left(10^{-14} \mathrm{~s}\right)$ in the solid, the transit of the energetic atom through the crystal until its stopping during which the electrons and excitation of the host atom and its environment subside, is $10^{-7}$ to $10^{-6} \mathrm{~S}$ [23].
Also, it has been observed that when amorphous non irradiated As2 $\mathrm{S}_{3}$ is annealed at the glass-transition temperature the medium-range order appears or increases, lessening the covalent bonding and increasing the chemical order [24]. Such an event was also seen on bombardment of amorphous $\mathrm{ZrO} 2$ with $\mathrm{Kr}^{+}$ions a small crystalline area is formed at the begining [25].

In this regard, referring to changes in valence states in the irradiated arsenic thiocompounds internal conversion (IC) phenomena should occur in huge number [26] 26generating vacancies cascades and producing a large amount of free electrons that is, a reductive ambience for recoil/excited atoms and ions, formation of $\mathrm{F}$ centers, reduction of $\mathrm{V}$ centers etc. On irradiated As2 3 , it is safe to postulate a similar mechanism to that of activated $\mathrm{Sb}_{2} \mathrm{~S}_{3}$, AsIII hot atoms could result by IC in the formation of ${ }^{*} \mathrm{AsV}$ and ${ }^{*} \mathrm{AsIV}$. The As IV valence state behaves [27] as a radical with a $\mathrm{d}^{10} \mathrm{~s}^{1}$ configuration, which dissociates into AsIII and AsV. The activation could probably forms both, the IV or $\mathrm{V}$ valence states. However, in the irradiated compounds, the As IV disproportionates in the crystal into AsIII and AsV increasing the yield of *As III and decreasing that of *As V.

Thermal annealing experiments, carried out at $80 \& 120 \pm 1^{\circ} \mathrm{C}$, confirm that of above exposed as it is shown in Fig 1. It is also shows that the *AsIII reach its final state in a short lapse after irradiation.

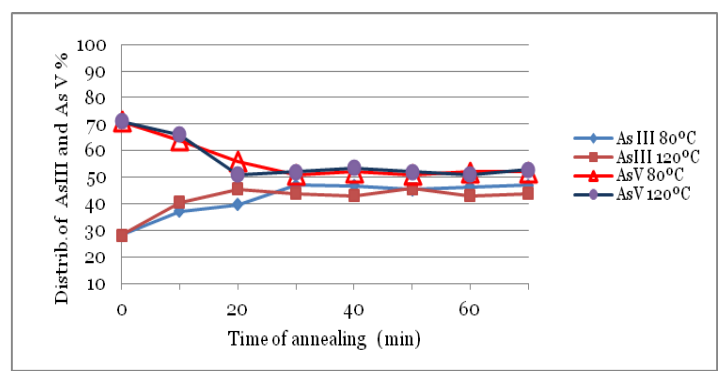

Figure 1. Annealing behavior of *AsIII \& *As V distribution irradiated As2 S3

These are assisted by large by the concentration/ availability of electrons and F centers in those defects created in the irradiated material; in $\mathrm{As}_{2} \mathrm{~S}_{5}$ the final state is reached immediately after the end of irradiation [28].

\section{CONCLUSION}

The higher yields of radioactive AsV in the sulfides, as compared to the oxides, can be attributed to the type of structure and to the higher covalent character of the As-S bond.

As per annealing behavior, in $\mathrm{As} 2 \mathrm{~S}_{3},{ }^{*} \mathrm{AsV}$ atoms are reduced to *AsIII by the large availability of electrons and $\mathrm{F}$ centers decreasing the yield of the former and increasing that of the latter; the final yield is reached a few minutes after the end of irradiation. In As2 55 the retention is $\sim 100 \%$ and the hot-atoms reach their final state during or immediately at the end of irradiations. 


\section{REFERENCES}

1. S. Radescu, A. Mujica, P. Rodríguez Hernández, A. Muñoz, J. Ibáñez, J.A. Sans, V.P. Cuenca-Gotor, F.J. Manjón, "Study of the orpiment and anorpiment phases of As2S3 under pressure", Journal of Physics: Conference Series, vol. 950: 42018, 2017. https://doi.org/10.1088/1742-6596/950/4/042018

2. V.P. Cuenca-Gotor, J.Á. Sans, O. Gomis, A. Mujica, S. Radescu, A. Muñoz, P. Rodríguez-Hernández, E.L. da Silva, C. Popescu, J. Ibañez, R. Vilaplana, F. J. Manjón; "Orpiment under compression: metavalent bonding at high pressure", Physical Chemistry Chemical Physics, vol. 22, no. 6, pp. 3352-336923, Jan. 2020. https://doi.org/10.1039/c9cpo6298j

3. V.P.Cuenca-Gotor, "Estudio de compuestos $\mathrm{As}_{2} \mathrm{X}_{3}$ bajo presión". Tesis doctoral dirigida F. J. Manjón, J. Á. Sans-Universitat Politécnica de Valencia, 311 pages, Jun. 2019.

4. I. Fejes, F. Billes, V. Mitsa, "A theoretical study of the effect on the vibrational spectrum of the stepwise sulfur by selenium substitution in arsenic pentasulfide", Journal of Molecular Structure THEOCHEM, vol. 531, pp. 407-414, Oct. 2000.

5. H. Kobayashi, H. Kanbara, M. Koga, "Third-order nonlinear optical properties of $\mathrm{As}_{2} \mathrm{~S}_{3}$ chalcogenide glass", Journal of Applied Physics, 74: 3683, 1993. https://doi.org/10.1063/1.354511

6. M. Wuttig, V.I. Deringer, X. Gonze, C. Bichara, J.Y. Raty, "Incipient Metals: Functional Materials with a Unique Bonding Mechanism", Advanced Materials, vol. 30, no. 51: 1803777, 2018 https://doi.org/10.1002/adma.201803777

7. N.R. Ramakant, K. Ganesan. S. Sangunni, "Optical properties change in amorphous (As2S3)o.87Sbo.13 thin films by photo and thermal induced process", J. Mater. Chem. Phys., vol. 125, no. 3, pp. 505-509, 2011. https://doi.org/10.1016/j.matchemphys.2010.10.025

8. A. G. Maddock and MM de Maine, "A search for the chemical effects of internal conversion following radiative neutron capture" Can. J. Chem, vol. 34, no. 4, pp. 441-444, 1956.

9. J.F. Facetti-M., "Chemical effects of neutron irradiation of antimony compounds", J. Inorg. Nucl. Chem., vol. 25, pp. 759-762, 1963.

10. G. Baró, A. Aten, "Chemical state of arsenic formed by nuclear transformations in the oxides of germanium and selenium", Proc. Symp. of Chem. Eff. of Nucl. Transf, IAEA, vol. 2, pp. 233-239, 1961.

11. H. Kawahara, G. Harbottle, "Thermal annealing of recoil arsenic atoms in neutron-irradiated solid compounds of arsenic", J. Inorg. Nucl. Chem., vol. 9, no. 3-4, pp. 240-245, 1959.

12. V. Kacena, A.G. Maddock, "The effects of compression on the annealing behaviour of neutron irradiated potassium dihydrogen arsenate crystals", Chemical Effects of Nuclear Transformations - IAEA Proceedings series, vol. II, pp. 255-262, 1965.

13. J. F. Facetti-M., E. Trabal, S. Torres, "Study of annealing of antimony compounds irradiated with neutrons", Primera Conferencia Interamericana de Radioquímica, Montevideo, pp. 234-236, 1965.

14. J.F. Facetti-M., H. Colmán, "Chemical effects of neutron capture in thioantimony compounds", J. Inorg. Nucl. Chem. vol. 33, pp. 419-423, 1971.

15. J.F. Facetti-M., A. Vallejos, "Chemical consequences of thermal annealing in neutron activated thioantimony compounds" J. Inorg. Nucl. Chem. vol. 34, pp. 3659-3664, 1972

16. Wen Li, "Synthesis and Solubility of Arsenic Trisulfide and Sodium Arsenic Oxy-sulfide Complexes in Alkaline Sulfide Solutions" MSc Thesis. The University of British Columbia, Vancouver, 123 pages, Jun. 2013.

17. R.B. Firestone, V.S. Shirley, Table of Isotopes, 8th ed. John Wiley \& Sons, New York, USA, 1996.

18. J.F. Facetti-M., H. Colman, A. Vallejos, "Separación por electroforesis de AsIII y As V en thiocompuestos de arsénico", Rev. Soc. Cientif. Paraguay, vol. 10, pp. 21-23, 1969.

19. A. F. Wells, Structural Inorganic Chemistry, Clarendon Press, Oxford, UK, 1984.

20. T.T. Meek, T.J. Isaacs, "Structure of amorphous bulk As2S5”, Journal of Electronic Materials, vol. 10, no. 4, pp. 653-664, 1981.

21. W.J. Stec, W.E. Morgan, R.G. Albridge, J.R. van Wazer, "Measured Binding Energy shifts of the " $3 \mathrm{p}$ " and " $3 \mathrm{~d}$ " electrons in arsenic compounds", Inorganic Chemistry, vol. 11, no. 2, pp. 216-225, 1972.

22. R.B. Firestone, "Adopted data base and user tables" in Database of Prompt Gamma Rays from Slow Neutron Capture for Elemental Analysis, IAEA, Vienna, ch. 7, pp. 73-75 \& 104-107, 2007.

23. G. Harbottle, "Hot Atom Chemistry in inorganic solids", Hot Atom Chemistry Status Report: Proceedings of a Panel, IAEA, Vienna, pp. 19-24, 1975.

24. K. Tanaka, "Chemical and medium-range orders in As2S3 glass", Physical Review B, vol. 36, no. 18, pp. 9746-9752, 1987.

25. G. Harbottle, "Effect of nucleogenesis preceding chemical reaction: dissipation of excitation before chemical reaction", in Chemical Effects of Nuclear Transformations in Inorganic Systems, G. Harbottle and A.G. Maddock, Eds., pp. 39-73, North Holland: Amsterdam, 1979.

26. T.A. Carson, "Primary processes in hot atom chemistry" in Chemical Effects of Nuclear Transformations in Inorganic Systems, G. Harbottle and A.G. Maddock Eds., pp. 11-38, North Holland: Amsterdam, 1979.

27. R.S. Nyholm, M.L. Tobe, "The stabilization of oxidation states of the transition metals in Advances" in Inorganic and Nuclear Chemistry and Radiochemistry, H. J. Emeleus and A.G. Sharpe Eds., vol. 5, p. 1, Academy Press: New York, 1963.

28. A. Vallejos, J. F. Facetti-M., "Chemical effects of neutron irradiation on germanium thiocompounds", Radiochim. Acta vol. 98, pp. 109-111, 2010. https://doi.org/10.1524/ract.2010.1688 